vol. 26 - $n^{\circ} 2 \mid 2010$

Numéro ouvert

\title{
L'état des recherches sur les associations de migrants internationaux
}

Migrants associations. A state of the art

El estado de las búsquedas sobre las asociaciones de emigrantes

\section{Antoine Dumont}

\section{(2) OpenEdition}

\section{Journals}

Édition électronique

URL : https://journals.openedition.org/remi/5147

DOI : 10.4000/remi.5147

ISSN : $1777-5418$

Éditeur

Université de Poitiers

Édition imprimée

Date de publication : 1 septembre 2010

Pagination : 117-137

ISBN : 978-2-911627-55-2

ISSN : 0765-0752

Référence électronique

Antoine Dumont, « L'état des recherches sur les associations de migrants internationaux », Revue européenne des migrations internationales [En ligne], vol. 26 - n² | 2010, mis en ligne le 01 septembre 2013, consulté le 14 avril 2022. URL : http://journals.openedition.org/remi/5147 ; DOI : https://doi.org/ 10.4000/remi.5147 


\title{
L'état des recherches sur les associations de migrants internationaux
}

\author{
Antoine Dumont*
}

es recherches sur les associations de migrants internationaux sont récentes en
France, puisqu'elles débutent après la loi du 9 octobre 1981 accordant aux étrangers le droit de s'associer. Paradoxalement, les premiers travaux portent surtout sur les associations "issues de l'immigration », celles regroupant des enfants de migrants, et sur leur rôle dans leur " intégration » à la société d'installation. En quelques années, l'élargissement de la sphère des associations étudiées permet un renouvellement des problématiques, avec les apports de la sociologie des relations interethniques et ceux des anthropologues et géographes travaillant sur les migrations africaines et les réseaux de développement créés par les migrants. Enfin, dans les années 2000, les recherches sur les associations de migrants tendent à s'européaniser.

Ce sont ces évolutions de la recherche que cet article se propose de retracer. Centrées au départ sur la notion d'intégration, ces études ont progressivement adopté le paradigme de l'ethnicité et l'ont exploré dans une perspective transnationale, en s'inspirant de travaux étrangers et en s'attachant à décrire les liens avec les espaces d'origine. Cet article aborde aussi, dans sa troisième partie, le débat sur la place et le rôle de l'État-nation vis-à-vis de ces appartenances autres exprimées par les associations de migrants. De fait, si cet état des travaux se veut aussi équilibré que possible, il accorde une plus large place aux lectures ayant nourri la thèse dont il est issu (Dumont, 2007). Les études sur le nationalisme et le sentiment national sont donc privilégiées ici, de même que les associations regroupant des migrants originaires d'un même État-nation. Enfin, l'approche proposée est critique, au sens où aucun des paradigmes examinés - l'intégration, l'ethnicité, voire le «transnationalisme »- ne permet de saisir l'ensemble des significations que les migrants accordent à leur origine nationale, lorsque celle-ci devient un moteur de leur engagement associatif dans l'espace d'installation.

* Docteur en géographie, membre associé de Migrinter, UMR 6588, Maison des Sciences de l'Homme et de la Société, Bât. A5, 5, rue Théodore Lefebvre, 86000 Poitiers, France ; antoine. dumont@univ-poitiers.fr 


\section{LES JALONS HISTORIQUES D'UN NOUVEAU CHAMP DE RECHERCHES}

Dans son travail sur les mobilisations de soutien à «la cause des sans-papiers », Johanna Siméant souligne le cloisonnement entre les recherches sur les migrations internationales et celles, plus rares, sur les actions collectives des migrants (Siméant, 1998 : 23). Dix années auparavant, dans la première thèse française sur les immigrés et la politique, Catherine Wihtol de Wenden avait déjà déploré la place marginale accordée aux migrants en science politique (Wihtol de Wenden, 1988 : 9). Jusqu'à la fin des années 1970, les immigrés sont en effet considérés comme des travailleurs temporaires, variables d'ajustement économique ou objets de politique publique, plutôt que comme des acteurs associatifs et politiques.

\section{Les associations de migrants avant 1981}

De 1939 à 1981, la loi française interdit aux étrangers de se constituer en association, mais l'historiographie atteste de mobilisations multiformes, en particulier durant les années $1970 .^{2}$ De fait, chaque nouvelle vague migratoire a produit son lot de regroupements par nationalités. L'exemple type est celui des Italiens, très actifs au sein de sociétés de secours mutuel puis d'associations antifascistes dans l'entre-deux-guerres et, après 1981, d'associations régionales à vocation économique ou culturelle. Parmi les autres nationalités ayant eu recours à ces regroupements, on citera les Polonais, les Espagnols, les Portugais et les Maghrébins (Wihtol de Wenden, 1988). Pour ces derniers, c'est la lutte pour l'indépendance des pays d'origine qui a motivé les regroupements les plus durables. Il en fut ainsi de l'Étoile nord-africaine, créée par l'Algérien Messali Hadj à Paris en 1926 (Sraïeb et al., 1985), ou de l'Association des Marocains en France (AMF), constituée en 1960 par Mehdi Ben Barka à Paris. Quant aux associations musulmanes, les premières émergent durant la seconde moitié du XIXe siècle et l'idée de créer une grande mosquée à Paris naît dès 1849 (Kepel, 1987 : 65). Ainsi, selon Rémy Leveau, la loi de 1981 est une "mesure symbolique aux effets politiques considérables» : symbolique, car durant la décennie 1970, "derrière chaque manifestation collective une association de fait existe déjà » (Leveau et Wihtol de Wenden, 1991 : 17-19). Enfin, bien avant 1981 et au-delà des groupes politiques ou culturels qu'ils constituent, les migrants entrent en contact avec de nombreuses associations de solidarité ${ }^{3}$ (Verbunt, 1980) tandis que l'extrême-gauche contribue à créer une « cause immigrée » aux marges du champ politique français (Siméant, 1998). Mais la recherche sur les mobilisations de migrants porte d'abord sur des aspects culturels, économiques et éducatifs, comme le montrent par exemple les sommaires de la revue Hommes \& migrations.

2 Les travaux d'histoire des migrations sont trop nombreux pour être cités ici. Nous rappellerons simplement qu'ils émergent dans les années 1980 et portent d'abord sur les migrations européennes (italienne, polonaise, etc.).

3 Notamment le Mouvement contre le racisme et pour l'amitié entre les peuples (MRAP, créé en 1949), la Fédération des associations de solidarité avec les travailleurs immigrés (FASTI, créée 1966) ou le Groupement d'information et de soutien aux travailleurs immigrés (GISTI, créé en 1972). 


\section{Les associations de migrants maghrébins après 1981}

Après 1981, les premiers travaux apparaissent sur les associations de migrants maghrébins et en particulier, sur celles créées par leurs enfants, qui ne sont souvent ni étrangers, ni migrants, mais dont les mobilisations sont liées à la prise de conscience d'une installation durable en France. Orientées vers l'égalité des droits et la lutte contre le racisme, ces mobilisations de jeunes sont d'abord interprétées en termes culturels, comme l'atteste la terminologie de l'époque : l'identité « beur» attribuée à l'ensemble des jeunes d'origine maghrébine serait le produit de leur " acculturation » (ici au sens d'assimilation culturelle) au sein de la société française. Cet accent mis sur la dimension identitaire des mobilisations permet de les inclure dans les «nouveaux mouvements sociaux" (Touraine et al., 1984). Le paradigme dominant est donc celui de l' « intégration », qui permet de distinguer les associations de jeunes «Beurs » de celles de leurs parents, réputées davantage orientées vers l'espace d'origine ou l'exercice du culte musulman. Les associations composant ce mouvement sont analysées dans une abondante littérature (Boubeker et Abdallah, 1993 ; Bouamama, 1994) proposant de nouvelles notions, telles «l'intégration conflictuelle » (Jazouli, 1986), "l'interculturalité » et la "seconde génération ». L'étude de ces associations emprunte à la sociologie de la jeunesse et aux travaux sur la « galère » (Dubet, 1987), la dimension ethnique n'étant abordée qu'incidemment ou tardivement (Boubeker, 2003). L'examen de la dimension musulmane de ces associations est réalisé dans une perspective géopolitique (Kepel, 1987) avant qu'elle devienne, dans les années 1990, un objet de sociologie politique (Cesari, 1998) ${ }^{4}$. Rares sont alors les auteurs qui défendent l'hypothèse d'Alain Battegay selon laquelle, avec le mouvement des "Beurs ", " une part d'un champ ethnique d'action militante s'est introduite dans l'espace médiatique français » (Battegay, 1990 : 112).

Avec le reflux du mouvement «beur » à la fin de la décennie 1980, l'échelle locale est privilégiée par des associations dont les revendications «civiques » s'effacent au profit d'activités soutenues par la politique de la ville, qui tend à délimiter l'espace associatif légitime (animation, soutien scolaire, lutte contre la délinquance, etc.). La recherche sur ces associations est dynamisée par la commande publique émanant de l'Agence de développement des relations interculturelles (ADRI) et du Fonds d'action sociale (FAS) (Leveau et Wihtol de Wenden, 1996), tout en empruntant progressivement des grilles de lecture critiques, qui insistent sur les logiques d'ethnicisation et de ségrégation à l'œuvre au sein de la société française et de ses institutions. À l'aube des années 2000, cette ethnicisation des rapports sociaux est plus souvent qualifiée de "post-coloniale ». Ainsi, dans un ouvrage de synthèse sur l'histoire des associations « beurs », Catherine Wihtol de Wenden et Rémy Leveau proposent l'hypothèse d'une réhabilitation dans les politiques publiques d'une gestion coloniale des populations immigrées, et s'interrogent : "La thématique de l'intégration serait-elle alors porteuse d'un idéal communautaire? La République créet-elle de l'ethnicité ? (Wihtol de Wenden et Leveau, 2001 : 123-124). Cette hypothèse, reprise notamment par Vincent Geisser (1997, 2005), est symptomatique des désillusions militantes et scientifiques suscitées par le déclin des associations « beurs ». Elle signifie

4 Dans sa postface à l'ouvrage d'Adil Jazouli (1986), le prêtre Christian Delorme, engagé dans le mouvement « beur », regrette l'absence de réflexion scientifique sur l'islam associatif et le rapport des jeunes à «l'islam des pères». 
aussi que l'opposition entre des formes intégrationnistes et communautaires de mobilisation était un modèle d'interprétation non scientifique, construit par les acteurs : militants, journalistes, responsables politiques, etc. Ainsi, «la façon dont la notion d'intégration est utilisée pour expliquer aussi bien la mobilisation que l'absence de mobilisation met en exergue son absence problématique de définition » (Siméant, 1998 : 41). Continuer à problématiser les questions en ces termes, c'est donc s'exposer à de nouvelles déconvenues. Ce point est aujourd'hui acquis par la recherche sur les associations de migrants, certains auteurs allant jusqu'à identifier en France des «formes d'État providence qu'on peut appeler "ethniques" » (Bordes-Benayoun et Schnapper, $2006: 202)^{5}$. En dehors du terrain "maghrébin », les chercheurs semblent avoir abandonné plus tôt l'opposition entre des formes « intégratives 》 et « communautaristes » de mobilisation. Les travaux sur les associations de migrants africains et portugais témoignent en particulier de l'attention accordée, dès les années 1990, au fonctionnement des réseaux associatifs, aux négociations identitaires et aux rapports avec les pays d'origine.

\section{Les associations de migrants portugais et africains}

Plusieurs recherches sont entamées au milieu des années 1980 sur les réseaux associatifs de migrants et sous l'impulsion de la commande publique. Par exemple, le rapport de Maurizio Catani et Salvatore Palidda, fruit du travail de vingt-six chercheurs, dresse un tableau général de ces réseaux tout en en proposant un solide cadre d'interprétation sociologique, dans lequel les migrants sont déjà qualifiés de «véritables acteurs dans un espace transnational » (Catani et Palidda, 1987 : 55). Par ailleurs, les travaux d'Albano Cordeiro (1986), de Michel Oriol et de Marie-Antoinette Hily (1985) sont riches d'enseignements sur les négociations identitaires au sein des associations de migrants portugais. En distinguant les mobilisations «instituées », qui favorisent des " contraintes formelles subies ou intériorisées » et les mobilisations « organisées » qui favorisent la « circulation de sens » entre les membres du groupe, ces auteurs soutiennent que ces associations ne sont pas seulement des «médiations entre les États et les communautés émigrées », mais aussi « des lieux de décentrement de la définition des appartenances» (Oriol et Hily, 1985 : 9). Le mouvement des «Beurs » peut être interprété ainsi : tout en proclamant leur fidélité aux origines familiales, les jeunes qui y participent instituent de tels lieux de médiation et de décentrement (associations, médias, groupes de musique, etc.). Dans le cas portugais, l'étude des associations participe désormais à celle, plus large, des relations des migrants avec l'espace d'origine (Kotlok-Piot, 1994), d'une « géographie de l'absence » (Poinard, 1991) et de la formulation d' "identités collectives à l'épreuve de la migration » (Hily, 1993). Ces liens avec l'espace d'origine et ces négociations identitaires sont également explorés auprès des associations de développement de migrants originaires de la région du fleuve Sénégal (Quiminal, 1991 ; Gonin, 1997 ; Daum, 1998). Au même moment, une autre évolution des problématiques apparaît, notamment dans d'autres travaux sur les migra-

5 Selon ces auteurs, " une partie des ressources publiques est accordée à des groupes particuliers pour qu'ils entretiennent leurs cultures. Bibliothèques, écoles juives ou arméniennes, associations culturelles ou folkloriques de toute origine bénéficient de subventions. On justifie cette aide par des arguments universalistes : il s'agit de maintenir une culture particulière, mais qui a une vocation universelle ; la reconnaissance publique des cultures d'origine rendra leur dignité aux populations d'origine étrangère et favorisera leur intégration à la société française » (ibid. : 202). 
tions africaines (Timera, 1997 ; Poiret, 1997) sur lesquels nous reviendrons, qui consiste à insister sur l'ethnicisation des rapports sociaux (De Rudder et al., 2000 ; Rinaudo, 2000) et à comprendre la place des migrants et de leurs associations dans la société française « au prisme de l'héritage colonial » (Blanchard et al., 2005). Cela étant, le discrédit attaché à l'origine ethnique demeure et décourage les migrants, comme les chercheurs, de la saisir " comme l'un des critères d'identification et de mobilisation collective » (ibid. : 243). Mais est-il pertinent d'établir l'ethnicité comme catégorie d'analyse des associations de migrants ? Et de quoi cette « ethnicité associative » serait-elle constituée ?

\section{LA PROBLÉMATIQUE CONTROVERSÉE DE L'ETHNICITÉ ASSOCIATIVE}

De nombreux travaux sur les migrations et les associations de migrants ont exploré cette problématique controversée de l'ethnicité associative. Avant de présenter certains d'entre eux, revenons sur la contradiction apparente entre association et ethnicité et sur les ambivalences constitutives de cette notion.

\section{L'ethnicité associative, une contradiction?}

En France, l'étude des associations de migrants a longtemps été centrée sur leur capacité à favoriser l'intégration à la société d'accueil. Sans revenir sur les débats autour de la notion d'intégration (Oriol, 1985 ; Dewitte, 1999 ; Hily et Rinaudo, 2002), nous rappellerons ici qu'elle est intimement liée à l'engagement associatif. En effet, elle est définie comme "la participation active à la société tout entière », y compris au sein d'associations témoignant de « spécificités culturelles» (HCI, 2000). Pourtant, quelques travaux persistent à distinguer entre les associations «communautaires » et celles « intégrationnistes ", selon leur public et leurs principes d'action (Baillet, 1998). Dans le langage des institutions, comme pour le sens commun, le contraire de l'intégration est le communautarisme ${ }^{6}$ qui, spatialisé, conduirait à la " ghettoïsation », forme extrême de la ségrégation ${ }^{7}$. Cette ghettoïsation de certains « quartiers sensibles » serait due, selon la police, à une «vie associative repliée, organisée en fonction de l'origine des partici-

6 Selon le Larousse 2004, le terme désigne « toute conception faisant prévaloir l'organisation de la société en communautés sur l'exigence d'assimilation des individus selon des règles et un modèle équivalent pour tous ».

7 La ségrégation peut être définie comme un processus de séparation tranchée qui affecte un groupe social ou un espace. Ce processus est marqué par une faible diversité sociétale, une forte discontinuité spatiale externe, une forte distanciation sociale et une qualification négative du groupe ou de l'espace concerné (Lévy et Lussault, 2003 : 831). 
pants $»^{8}$. Les associations de migrants sont donc réputées avoir un rôle dans leur intégration ou leur ségrégation au sein de la société d'installation. Toutefois, ce rôle n'est pas exactement celui qu'on voudrait leur faire jouer.

En effet, la sociologie a montré que la vie associative est composite et qu'à côté de l' « entre soi », de l' « être ensemble », existe un pôle plus offensif, le « faire ensemble ». Le modèle associatif français est un "modèle mixte », permettant à la fois la participation communautaire et l'intégration à la société et à l'État central (Ion, 1997 : 25-29), ainsi que la création d'associations "hybrides » mêlant sociabilités primaires et secondaires (Laville et al., 2001 : 192). Cette double orientation n'est donc pas propre aux associations de migrants, leur seule spécificité à cet égard étant peut-être de viser à recréer une "primarité » vécue dans l'espace d'origine. Ne serait-ce pas justement cette spécificité « ethnique » qui singulariserait les regroupements de migrants dans le champ associatif français ? Si toutes les associations sont fondées, pour partie au moins, sur les appartenances particulières de leurs membres, la controverse du communautarisme ne vientelle pas du fait que ces appartenances sont, dans le cas des migrants associés, de nature ethnique ? Pour y répondre, il faut revenir brièvement sur la notion d'ethnicité et sur ses ambivalences en sciences sociales.

\section{L'ethnicité en sciences sociales : une notion ambivalente}

Si Max Weber qualifiait d'ethniques les « groupes humains qui nourrissent une croyance subjective à une communauté d'origine fondée sur des similitudes de l'habitus extérieur ou des mours, ou des deux, ou sur des souvenirs de la colonisation ou de la migration » (Weber, 1971 : 416), au début du XXe siècle le mot « ethnie » est surtout employé par les ethnologues - soviétiques entre autres - comme substitut au terme de race (Gossiaux, 2002 : 7-10). Cet usage substantialiste de la notion, en négligeant les changements culturels internes aux groupes, aboutit à une naturalisation du phénotype, de la parenté de sang, voire de la langue, de la religion ou de la coutume. Cette substitution du terme d'ethnie à celui de race est donc critiquée dans les années 1970 pour entretenir la confusion entre différences culturelles et caractéristiques physiques, notamment dans les recherches sur les migrations internationales (Guillaumin, 1972 : 58-60). Les ethnic studies opèrent alors un glissement du "groupe ethnique " à la "minorité ethnique ", celle porteuse d'une culture étrangère exprimée de manière collective, associative par exemple. Peu à peu, l'ethnicité est pensée comme constitutive des rapports de domination entre majoritaires et minoritaires : pour les premiers, "l'ethnicité c'est l'humanité des autres » (Simon, 1994 : 19) et ils attribuent aux seconds une ethnicité particulière. Ainsi, dans toute société, " c'est le fait d'être exclu de l'état commun et, en particulier, d'être

8 Citation d'un rapport de 2004 de la Direction centrale des renseignements généraux. Selon ce rapport, repris en partie par Le Monde du 6 juillet 2004, plus de 300 quartiers regroupant près de deux millions de personnes seraient "ghettoïsés ou en voie de l'être ». Les facteurs de " ghettoïsation » seraient surtout culturels : "repli sur la culture d'origine ", "rejet des valeurs occidentales », etc. Ainsi, "ces populations conservent des survivances culturelles aboutissant à une certaine endogamie, à un maintien des modes de vie traditionnels, à l'émergence de modes de régulation sociale des conflits parallèles aux institutions, à une vie associative repliée, organisée en fonction de l'origine des participants » (souligné par moi). 
exclu du pouvoir partagé, qui est ethnicisé » (Gossiaux, 2002 : 41-42). Le majoritaire n'y est jamais qualifié d'ethnique, car « ce qui prend rang de marque est réservé au minoritaire » (Guillaumin, 1972: 77) ${ }^{9}$. Du point de vue du sociologue, les majoritaires sont bien entendu aussi « ethniques » que les minoritaires, au sens où ils produisent de l'ethnicité. Ainsi, on peut affirmer avec Danielle Juteau-Lee (1983 : 51) qu' « être humain, c'est être ethnique » et que l'ethnicité est d'abord produite par le travail d'éducation des enfants, par les femmes le plus souvent.

Ce qui précède ne saurait faire oublier que le véritable tournant épistémologique date de 1969, avec la publication du texte de Fredrick Barth ${ }^{10}$. Sa définition des groupes ethniques part du constat de la persistance des frontières ethniques malgré leur franchissement par les individus et l'existence de « relations sociales stables, persistantes et souvent d'une importance sociale vitale » à travers ces frontières (Poutignat et StreiffFenart, 1995 : 204). Pour Fredrick Barth, l'ethnicité est une catégorie d'identification qui classe une personne «selon son identité fondamentale, la plus générale, qu'on présume déterminée par son origine et son environnement » (ibid. : 210-211). Cette identité fondamentale réunit des traits culturels que les acteurs - membres du groupe désigné ou non considèrent comme significatifs et qui deviennent des « signes manifestes » d'ethnicité ${ }^{11}$. Les traducteurs français d'Ethnic groups and boundaries reformulent ainsi la définition barthienne : "l'ethnicité est une forme d'organisation sociale, basée sur une attribution catégorielle qui classe les personnes en fonction de leur origine supposée, et qui se trouve validée dans l'interaction sociale par la mise en ouvre de signes culturels socialement différenciateurs » (ibid. : 155). Cette définition contient les idées d'attribution catégorielle et d'origine supposée, d'inspiration weberienne, qu'elle fait tenir ensemble en insistant sur le caractère dynamique et interactionniste de l'ethnicité : ce n'est donc pas le contenu culturel d'un groupe qui importe, mais les limites qu'il trace vis-à-vis des autres groupes - et réciproquement - en puisant certains éléments dans ce contenu culturel. Cette mise en scène de signes culturels socialement différenciateurs peut être qualifiée de conscience ethnique. Sans conscience ethnique ressentie par les individus, on ne peut parler de groupe ethnique. Par contre, cela n'empêche pas d'autres acteurs sociaux de leur attribuer une identité ethnique, c'est-à-dire de les ethniciser.

C'est à partir de ce texte fondateur que les ethnic studies se développent (de la manière qui a été indiquée plus haut) et qu'elles sont introduites en France, au milieu des années 1980, dans les recherches sur les migrations internationales (Guillon et TaboadaLeonetti, 1986). Dix ans plus tard, elles feront l'objet d'un traitement sémantique, avec la

9 «Les caractères physiques du majoritaire n'ont pas le même statut de marque, en effet ils ne sont pas destinés à être des limites ni des spécifications. [...] Ce qui prend rang de marque est réservé au minoritaire et ne prend sens que dans son rapport à ce qui n'est pas marqué ; la race prend son sens dans ce qui n'est pas racisé » (Guillaumin, 1972 : 76-77). L'auteur note toutefois des exceptions de racisation d'eux-mêmes par les dominants, tel l'aryanisme des XIX et XXe siècles.

10 Écrit en 1969, «Ethnic groups and boundaries » a été traduit en français en 1995 (Poutignat et Streiff-Fenart, 1995 : 203-249). Les citations et leur pagination se rapportent à cette version française.

11 Ces signes peuvent être « le costume, la langue, l'habitat, ou le style de vie en général », ou des « orientations de valeurs fondamentales : les critères de moralité et d'excellence par lesquels les actes sont jugés » (ibid. : 211). 
publication en 1994 d'un Vocabulaire historique et critique des relations interethniques, de travaux de traduction et de synthèse théorique (Poutignat et Streiff-Fenart, 1995 ; Martiniello, 1995). Au fil du temps, des terrains nouveaux sont explorés, par exemple sur l'entreprenariat ethnique (Ma Mung, 1992), et des débats inédits sont ouverts, tel celui sur l'usage de « catégories ethniques » (Simon, 2003). Surtout, l'idée d'une ethnicité associative commence à émerger dans les études sur les diasporas.

\section{L'ethnicité associative dans l'étude des diasporas}

Ces études affirment le rôle joué par les associations de migrants dans la structuration diasporique d'un groupe migrant et le fonctionnement de ses réseaux transnationaux. Les actions associatives contribuent en effet à la formation de mémoires collectives diasporiques (Bruneau, 2004 : 155, 192). Outre le cas des réfugiés grecs d'Asie mineure décrit par Michel Bruneau, d'autres exemples sont développés à propos de la mémoire antillaise liée à l'esclavage (Chivallon, 2002) ou de la mémoire mexicaine lors de la migration aux États-Unis (Faret, 2003). Dans ces exemples, les associations sont bien identifiées comme des vecteurs de l'ethnicité des migrants, aux côtés des pratiques culturelles, religieuses et linguistiques (Bruneau, 2004 : 155). D'autres auteurs vont plus loin en accordant davantage de place aux associations dans leurs observations et à l'ethnicité associative dans leur conceptualisation.

Le premier exemple en est l'ouvrage d'Albert Bastenier sur la « société ethnique 》 définie comme « celle qui organise socialement la conflictualité entre les différentes identités socioculturelles » (Bastenier, 2004 : 6). Battant en brèche le paradigme de l'intégration, il décrit la marche pour l'égalité de 1983 comme une transgression des frontières ethniques ayant échoué en raison de l'absence d'associations représentatives (ibid. : 269). Les notions de réseaux transnationaux et de décentrement identitaire sont convoquées (ibid. : 242), de même que l'ethnicisation de la société mise en relation avec le changement d'échelle engendré par la globalisation et la complexification des flux migratoires (ibid. : 143, 161). Pour autant, deux questions demeurent de notre point de vue. D'une part, les exemples cités se limitent souvent aux associations musulmanes et à ce que l'auteur nomme le "matériau culturo-religieux" de leur conscience ethnique, tout en réservant «le matériau culturo-politique sécularisé de la nationalité » aux seuls majoritaires. Les migrants n'ont-ils pas une nationalité ? Que deviennent les consciences nationales en migration? Ces questions sont au cœur d'une série de travaux de science politique présentés plus loin. D'autre part, en définissant l'ethnicité comme une organisation sociale des différences culturelles (ibid. : 100) et en la proposant comme paradigme central, Alain Bastenier s'expose au risque d'une ethnicisation des sciences sociales qui négligerait les logiques de domination, alors même que l'ethnicité peut être pensée comme relevant d'une logique formelle pauvre selon laquelle « un individu ne peut appartenir qu'à un seul groupe ethnique » (Gossiaux, 2002 : 17).

Le second exemple, quoiqu'antérieur, est l'ouvrage de Christian Poiret (1997) qui décrit finement les logiques d'ethnicisation des migrants africains et de leurs associations en France. Ce processus s'expliquerait par le déclin du mouvement ouvrier et par le rôle des acteurs institutionnels, même si les situations varient selon les territoires considérés. Ainsi, «à l'origine de chacune des associations africaines territorialisées [qu'il a rencon- 
trées], il y a quelques acteurs institutionnels » qui les encouragent et les subventionnent (Poiret, 1997 : 393), car ils jugent pertinente la catégorie ethnique « Africain », pour de multiples raisons ${ }^{12}$. À la même époque, on retrouve dans les travaux de Mahamet Timera (1997) sur l'ethnicisation des migrants africains la même attention accordée à l'histoire des populations migrantes, aux contextes locaux de leur installation et aux stratégies des acteurs associatifs et institutionnels. Ce dernier aspect, essentiel, était déjà développé dix ans plus tôt dans un article de Michel Giraud et Claude-Valentin Marie (1987), qui interprétait le renforcement des solidarités communautaires et l'affirmation d'une identité ethnique comme une stratégie des associations antillaises de métropole dans leurs rapports avec l'État français. La même idée était au cœur d'un travail de Catherine Quiminal sur les associations de migrants soninké, pour lesquelles « cette dynamique ethnique sert de monnaie d'échange dans le champ des relations avec la société française » (Quiminal, 1991 : 214).

\section{Ethnicité associative, citoyenneté et territoire}

Cet intérêt renouvelé, dans l'étude des associations de migrants, pour les contextes locaux et les relations avec les États d'origine et d'installation souligne la justesse d'une remarque de Christian Poiret (1997 : 27) : «depuis une quinzaine d'années, le centre de gravité des relations entre immigrés et mouvement ouvrier s'est déplacé du terrain de l'entreprise vers celui du territoire et de la citoyenneté ». Le mouvement «beur» avait déjà ouvert une réflexion sur ces formes alternatives de citoyenneté (Bouamama et al., 1992) et, dans les années 2000, les chercheurs ont continué à s'intéresser aux manières dont, dans différents espaces, des associations " ethniques » exercent et revendiquent l'exercice de la citoyenneté. La série de travaux présentée dans ce paragraphe illustre cette tendance de la recherche, en même temps qu'elle souligne en creux les spécificités du contexte français.

Aux Pays-Bas, l'étude des associations de migrants menée par l'Institute for Migration and Ethnic Studies (IMES) est centrée autour de la notion de « communauté ethnique civique » (ethnic civic community). Proche de l'idée d'ethnicité associative, cette notion a été forgée dans le cadre d'une analyse des réseaux formés par les associations de migrants d'une même origine nationale dans des villes d'Europe occidentale (Fennema et Tillie, 2004). Ces auteurs mesurent la cohésion de ces réseaux par deux indicateurs : la densité organisationnelle, soit le nombre d'associations rapporté à la population du groupe considéré, et la densité du réseau, égale au nombre de relations existantes entre ces associations divisé par le nombre de leurs relations potentielles ${ }^{13}$. Le point essentiel est le lien établi entre cette densité et le caractère civique de la communauté ethnique ainsi

12 Notamment la concentration spatiale de la population ainsi désignée, les possibilités de contact direct avec elle, les problèmes qu'elle rencontre et l'interpellation par des acteurs qui en sont issus (ibid.).

13 Une relation entre deux associations existe quand un même individu est membre de leurs bureaux respectifs. Ainsi, la densité du réseau associatif s'accroît avec la multi-appartenance. Par exemple, dans une ville, le nombre d'associations de Marocains (n) est de huit et l'on observe quatre relations entre elles $(z=4)$. Le nombre de lignes potentielles $(y)$ est de vingt-huit, car $y=n(n-1) / 2$. La densité du réseau (d) est donc de 0,14 , car $\mathrm{d}=\mathrm{z} / \mathrm{y}$. 
organisée. Ce lien est constaté à travers les taux de participation électorale et expliqué par la diffusion de la confiance entre migrants associés et par la nature démocratique des associations. Ainsi, l'augmentation du nombre d'associations de migrants ne serait pas le signe d'une intégration politique défaillante, mais précisément le contraire : plus la communauté ethnique civique est forte, plus ces membres participent politiquement, plus ils ont confiance dans les institutions démocratiques. Cet argument est sans doute moins valable dans le cas français, tant 1' « assimilationnisme civique » s'oppose au "pluralisme civique » néerlandais (Koopmans et Statham, 2001 : 74-75). L'usage de la notion de "communauté ethnique civique » doit donc être soucieux des différents contextes nationaux.

Cette précaution est suggérée par Meindert Fennema et Jean Tillie lorsqu'ils posent, parmi les cinq facteurs expliquant l'émergence d'associations de migrants, la structure des opportunités politiques de l'État d'installation et l'influence transnationale de l'État d'origine (Fennema et Tillie, 2004 : 88). Le premier a déjà été appliqué aux mobilisations de migrants en France (Siméant, 1998) et nous reviendrons sur le second dans la troisième partie de cet article. Les trois derniers facteurs mentionnés sont l'existence d'attributs ethniques, le niveau des ressources et les trajectoires et lieux d'installation des migrants (Fennema et Tillie, 2004 : 88). Ce dernier facteur, spatial, est en fait négligé par les travaux inspirés de la notion de "communauté ethnique civique ». Un changement d'échelle d'observation, de l'espace urbain à l'espace de vie transnational des migrants, conduirait sans doute à des résultats différents quant au «civisme » du groupe étudié. De fait, rien n'est dit des relations entre associations localisées dans différentes villes, a fortiori dans différents pays. La mobilité spatiale individuelle n'est pas non plus considérée, par exemple comme facteur favorisant la multi-appartenance associative, et le rapport de ces associations avec l'espace d'origine n'est pas exploré, à travers notamment des pratiques de circulation migratoire.

Malgré leurs limites, ces travaux néerlandais ont le mérite de montrer que s'associer selon son origine n'est pas incompatible avec l'exercice d'une citoyenneté dans l'espace de résidence. L'attention portée aux configurations militantes locales rejoint des études françaises déjà mentionnées. Celle sur le rôle des associations dans la cohésion communautaire conforte les acquis des études sur les diasporas. Pour autant, la pertinence d'une approche en termes d'ethnicité associative reste à démontrer. S'agissant des associations basées sur l'origine nationale de leurs membres, ne serait-il pas judicieux de raisonner d'abord en termes de nationalité, de sentiment d'appartenance ? Dans cette hypothèse, l'ethnicité serait considérée comme l'une des catégories (re)produites par les acteurs, dont rien n'indique a priori qu'elle domine les autres. On remarquera d'ailleurs que les migrants, désignés comme ethniques dans les sociétés d'installation, sont considérés comme des nationaux lorsqu'ils sont à l'intérieur des frontières de l'État dont ils sont originaires. La question de la mobilité spatiale des individus et de l'inscription territoriale de leurs mobilisations doit donc être explorée. Pour cela, cet état des travaux doit s'élargir dans un dernier temps à ceux s'intéressant à la dissociation entre l'État, la nation et le territoire et aux transformations du nationalisme dans un espace transnational. 


\section{LES RECHERCHES SUR LA NATIONITÉ ASSOCIATIVE ET L'ESPACE TRANSNATIONAL}

Cette perspective théorique est balisée par des travaux en géographie et en science politique qui portent sur la transnationalisation ${ }^{14}$ des mobilisations et des sentiments d'appartenance. Divers dans leurs orientations et leurs méthodes, ces travaux ont pour point commun de libérer l'étude des associations de migrants de l'emprise du cadre national, en intégrant des définitions et des problématiques forgées ailleurs dans le monde. Nous insisterons ici sur deux des effets de ce desserrement, dans la recherche française, de l'emprise du cadre national : l'un a trait à l'articulation entre la nationalité et l'ethnicité, l'autre concerne la déconnexion entre le nationalisme et le territoire.

\section{L'articulation entre la nationalité et l'ethnicité : la nationité associative}

La dissociation théorique effectuée par les politistes entre les concepts d'État, de nation et de territoire ${ }^{15}$, qui se fonde sur leur observation des phénomènes sociaux inscrits dans la globalisation ${ }^{16}$, a permis de renouveler les approches historiques et sociologiques de l'État-nation et du nationalisme. Celui-ci est défini par Ernest Gellner comme une idéologie née de la modernisation industrielle et de l'homogénéisation culturelle, dont l'objectif est de faire coïncider limites ethniques et limites politiques, autrement dit de superposer le territoire de l'État à celui de la nation, définie par son unité culturelle (Gellner, 1989). D'autres historiens complètent cette définition en montrant que, si le nationalisme précède la nation, celle-ci ne procède pas d'une ethnie pré-existante. Des «protonations » peuvent certes exister, mais la plupart ne sont pas devenues - et ne deviendront pas - des nations au sens d'État-nations (Hobsbawm, 1992: 100). En ce sens, «très peu de mouvements nationaux modernes trouvent leur origine effective dans une forte conscience ethnique » (ibid. : 86) ${ }^{17}$. Dans le même esprit, Benedict Anderson (2002) insiste sur le rôle des élites et des techniques et qualifie la nation de "communauté imaginée » formée par le « capitalisme de l'imprimé » et, plus largement, par le progrès technique permettant «l'interchangeabilité » de fonctionnaires de plus en plus mobiles. Le nationalisme ainsi défini, résultat d'un long processus historique, n'est donc pas la simple traduction,

14 Ce terme est préféré à celui de transnationalisme. Il désigne simplement ici la construction de réseaux sociaux durables par-delà les frontières des États-nations. En géographie, ce terme est parfois remplacé par celui de «transnationalité ».

15 Le mot est employé ici dans son sens restreint : il désigne un espace borné, régulé et contrôlé par un groupe social, dont le pouvoir s'exerce par la médiation du sol. Cette définition est inspirée de Bertrand Badie (1995 : 11-12).

16 Définie comme un processus d'interdépendance croissante des lieux entre eux à l'échelle mondiale. Jacques Lévy souligne que ce processus (qu'il nomme mondialisation) oblige à «renégocier des compromis sociaux établis dans le cadre national » (Lévy et Lussault, 2003 : 639).

17 Éric Hobsbawm distingue le nationalisme moderne du «proto-nationalisme populaire », fondé sur la langue, l'ethnie, la religion, la tradition dynastique et la conscience d'appartenir ou d'avoir appartenu à une entité politique. Mais il distingue également ce nationalisme moderne, dont l'apogée se situe de 1918 à 1945, des mouvements en faveur de l'indépendance des pays colonisés et du « réveil ethnique » de la fin du XXe siècle. 
dans la sphère politique, d'une ethnicité pré-existante. Si le nationalisme constitue bien l'un des socles idéologiques de l'État-moderne et des concepts modernes de nation et de nationalité, l'autre socle est le libéralisme philosophique et politique du XIXe siècle. C'est ce qu'explique Christophe Bertossi, pour qui «l'élément déterminant qui distingue nationalité et ethnicité tient au fait que la nationalité participe directement du processus de modernisation des appartenances politiques, à mettre à l'actif du nationalisme libéral dans le siècle qui suit les révolutions américaine et française » (Bertossi, 2001 : 49).

Dans une synthèse récente des théories du nationalisme, Christophe Jaffrelot souligne que Gellner et Anderson semblent confondre sentiment national et nationalisme (Dieckhoff et Jaffrelot, 2006 : 41). Il reprend en cela la critique de Pierre Birnbaum leur reprochant de trop s'attacher à déconstruire le mythe national sans expliquer la force du sentiment d'appartenance à la nation (Birnbaum, 1997 : 27-28). Selon ces auteurs français, les théories du nationalisme gagneraient aujourd'hui à emprunter aux théories de l'ethnicité, non pas pour qualifier certaines formes de nationalisme d' "ethniques » ou pour dire que l'ethnie précède la nation, mais pour insister sur le rôle des conflits d'intérêts entre différents groupes dans leur rapport à l'État et dans la définition de leurs frontières réciproques, que ces groupes soient qualifiés de nationaux ou d'ethniques (Dieckhoff et Jaffrelot, 2006 : 102-103). Cette suggestion n'est pas nouvelle ; elle fut formulée notamment par Claudie Weill (1987 : 45) dans son travail sur les relations interethniques dans la IIe Internationale. Appuyée sur la définition barthienne de l'ethnicité, elle nous semble toujours justifiée et surtout très pertinente pour les recherches sur les associations de migrants. Ainsi, les identités collectives portées par ces associations dans les espaces d'installation peuvent être qualifiées d'ethniques, de nationales, ou tout autrement selon les cas.

Dès lors, plutôt que d'abandonner l'idée d'ethnicité associative, il convient de la refonder sur ce qui est au fondement de nombreuses associations de migrants, à savoir leur attachement à leur origine nationale, leur sentiment d'appartenance nationale. C'est ce sentiment que Benedict Anderson nomme la nationité (nationness), en l'opposant à la nationalité (nationality) : la seconde ouvre l'accès aux droits politiques, tandis que la première désigne le sentiment d'appartenir à une "communauté nationale » définie par une histoire, une culture, une religion et une langue (Anderson, 2002). Cette distinction permet de comprendre que la possession de la nationalité d'un État ne s'accompagne pas toujours d'un sentiment d'appartenance à la nation représentée par cet État. Et même lorsque c'est le cas, la nationalité à elle seule ne garantit pas la reconnaissance sociale d'une telle identification (Neveu, 1993 : 287). Ce n'est donc pas l'ethnicité associative, mais la "nationité associative » qui devient le cœur de nos interrogations. L'ethnicité associative et le «nationalisme associatif » sont seulement deux formes que peut prendre la nationité associative. Mais, affirmant cela, la recherche aurait-elle avancé ? Qu'apporte l'idée de nationité associative à l'étude des associations de migrants originaires d'un même pays ? Pour y répondre, il faut intégrer à la réflexion un dernier élément : la distance qui sépare ces migrants de leur espace d'origine.

En effet, étudier et conceptualiser la nationité associative des migrants nécessite non seulement de croiser les théories de la nationalité et de l'ethnicité, mais aussi de tenir compte du fait que cette nationité s'exprime à distance, à partir d'un autre État que 
celui auquel cette nationité est liée. C'est ce dont rendent compte des notions récemment proposées telles que le «nationalisme de longue distance » (Anderson, 1998 ; Glick Schiller et Fouron, 2002) et le «nationalisme transnational » (Kastoryano, 2006). Selon Nina Glick Schiller, la première désigne un ensemble de pratiques et de revendications identitaires reliant des personnes qui affirment descendre d'un pays ancestral (Glick Schiller, 2004 : 459). La notion de nationalisme transnational désigne quant à elle un "nationalisme sans territoire " formulé par des élites migrantes. Riva Kastoryano la construit en l'opposant en partie au «nationalisme diasporique » décrit par Gellner (1989). Celui-ci désignait ainsi la revendication d'un territoire, au début du XXe siècle, par des groupes «parias » (Juifs, Arméniens, Grecs), dont l'impuissance politique aurait été révélée par l'industrialisation. Le nationalisme transnational diffère de celui de ces diasporas sur trois points : il apparaît parmi des migrants économiques post-coloniaux plutôt que des exilés d'un territoire d'origine ; il vise à l'assimilation institutionnelle dans l'État d'installation plutôt que la construction d'un nouvel État-nation ; enfin, il promeut l'idée d'une autonomie personnelle et culturelle non-territoriale, plutôt qu'une auto-détermination politique et une reconnaissance dans le système international (Kastoryano, 2006 : 537-541). Cette notion est pertinente, car elle postule que le nationalisme est à la fois une idéologie fortement territorialisée et un sentiment d'appartenance à une collectivité qui peut s'exprimer à distance. L'expression contradictoire de «nationalisme transnational » traduit bien le fait, largement démontré, que les réseaux transnationaux peuvent renforcer le nationalisme (Dorais, $2004: 52$ ).

\section{La déconnexion entre le nationalisme et le territoire : l'espace transnational}

Qu'il soit diasporique, transnational ou de longue distance, le nationalisme ainsi conçu est découplé du territoire. En un sens, il s'agit d'un phénomène récent, conséquence de la crise de l'État-nation dans un contexte de globalisation des flux. Mais il s'agit aussi, sur le plan théorique, de la réactivation de conceptions formulées après 1905 au sein la IIe Internationale, notamment par les austro-marxistes, pour lesquels le territoire jouait un rôle secondaire dans la formation du nationalisme et de la nation moderne. Ce camp « extra-territorialiste » (Weill, 1981), opposé à celui, plus matérialiste, de la nation territorialisée (Kautsky, Staline, etc.), était bien représenté par Otto Bauer. Observant le réveil des «petites nations » de l'empire austro-hongrois, Bauer fondait sa définition de la nation sur l'histoire plutôt que sur les éléments matériels observables (territoire, langue, coutume, etc.) qui découlaient de cette « communauté de destin » permise par le partage d'une même expérience historique (Juteau, 1983 ; Weill, 1981, 1987). Un siècle plus tard, ce n'est plus de crise des empires multinationaux dont il est question, mais de celle de l'État-nation, qui reste diversement interprétée : certains auteurs y voient la " fin des territoires », au sens restreint (stato-national) qu'ils donnent à ce mot (Badie, 1995) ; d'autres, tenants du "post-nationalisme », y lisent les progrès de l'universalisation des droits, qui dissocierait la citoyenneté de la nationalité en favorisant l'émergence d'ensembles supra-nationaux (Soysal, 1994) ; enfin, les promoteurs du «transnationalisme » l'interprètent comme le signe d'une « déterritorialisation » des États-nations et de la formation d'espaces sociaux transnationaux (Basch et al., 1994). Par-delà leurs divergences d'interprétation, tous ces auteurs admettent que la communauté nationale n'est plus - et de moins en moins - la 
seule communauté politique signifiante sur la scène internationale : les sentiments d'allégeance des individus à l'égard d'États-nations en recomposition perdent de leur force et, partant, de leur potentiel de mobilisation, tandis que le territoire national devient un territoire parmi d'autres, qu'ils soient supra ou infranationaux. La nation n'est plus le seul cadre d'affirmation de la citoyenneté, qui s'exerce désormais sur différents espaces et à diverses échelles. Si nous partageons ces constats, les interprétations globales dans lesquels ils s'inscrivent ne nous semblent pas toujours à même d'expliquer la diversité, observée sur le terrain, des reformulations associatives de la nationité des migrants.

Ainsi, la thèse de Bertrand Badie, qui affirme que « le territoire est de moins en moins admis comme le support d'une identité politique citoyenne et de plus en plus toléré ou réclamé comme l'instrument d'une identité religieuse ou ethnique » (Badie, 1995 : 254), est discutable en fonction de la définition donnée au territoire. Des géographes s'appuyant sur une définition plus complexe du territoire montrent au contraire son «étonnant dynamisme » et concluent que "la mondialisation se prête particulièrement à un fort développement territorial » (Di Méo et Buléon, 2005 : 96, 78). Une telle approche permet en outre de desserrer un peu plus l'emprise du cadre national sur la réflexion scientifique.

La thèse "post-nationaliste », selon laquelle l'existence d'un cadre juridique supra-national permet aux migrants de se mobiliser en tant qu'êtres humains, au-delà de leurs appartenances nationales, pour obtenir la satisfaction de leurs revendications (Soysal, 1994) est-elle plus utile à la recherche sur les associations de migrants ? Outre qu'elle semble pécher par excès d'optimisme, cette thèse est très peu confirmée par des enquêtes de terrain. On lui reproche également de sous-estimer l'acculturation des migrants dans les sociétés d'installation et de ne pas contextualiser leurs revendications (Bordes-Benayoun et Schnapper, 2006 : 154). Enfin, l'existence de droits à l'échelle internationale n'implique en rien l'homogénéité de leur application par les États ou de leur appropriation par les migrants. Par exemple, les modèles nationaux de citoyenneté exercent toujours une forte influence sur les modes de mobilisation des migrants et les identités collectives qu'ils valorisent (Koopmans et Statham, 2001).

La thèse «transnationaliste » souligne au contraire l'hétérogénéité de la globalisation et, dans une approche interactionniste, accorde aux migrants le statut d'acteurs à part entière (Basch et al., 1994 ; Hannerz, 1996). L'attention portée aux reformulations identitaires, notamment associatives, se traduit par de nouvelles notions, telle celle d' " ethnoscape », ou paysage d'identité, qui prennent acte de la dissociation croissante entre les territoires stato-nationaux, les subjectivités et les mouvements sociaux (Appadurai, 2001 : 89, 260). Cependant, par-delà la diversité des travaux qui s'en inspirent (Faist, 2004 ; Levitt, 2001 : Smith ; Guarnizo, 1998 ; Smith, 2001), la thèse transnationale semble souffrir de trois lacunes lorsqu'on tente de l'appliquer à un objet précis. Les principaux auteurs des transnational studies appliquées aux migrations en sont conscients et cherchent à délimiter ce champ de recherche, qui couvre un ensemble très hétéroclite d'objets (Glick-Schiller, 1997 ; Portes et al., 1999 ; Vertovec, 1999). Notre propos n'est pas de résumer le leur, mais de dégager trois points qui peuvent encore poser problème, s'agissant des concepts d'État déterritorialisé et d'espace transnational appliqués à l'étude des associations de migrants. 


\section{Au-delà du «transnationalisme » : quelques pistes à explorer}

Le premier problème réside dans la notion d' "État-nation déterritorialisé », qui cherche à qualifier le maintien des liens d'allégeance des migrants à leur État d'origine ; autrement dit, qui traduit l'idée selon laquelle là où des migrants se trouvent, on trouve l'État d'origine (Basch et al., 1994 : 269). Mais l'expression est contradictoire : d'une part, tout État par définition exerce son autorité sur un territoire et on voit mal ce que serait la légitimité d'un État sans territoire ; d'autre part, qu'il s'agisse d'une organisation politique ou sociale, toute déterritorialisation s'accompagne par définition d'une reterritorialisation. Ces deux postulats, appuyés sur les définitions classiques de l'État en science politique et de la territorialisation en géographie, interdisent l'usage de la notion d' « État déterritorialisé » au profit de notions moins controversées, telles celles de "citoyenneté flexible », de «zones de souveraineté graduelles » des États d'origine sur «leurs » migrants (Ong, 1999). À l'inverse, cela n'interdit pas de chercher à comprendre comment les associations transnationales de migrants contribuent au renouvellement des modèles nationaux de citoyenneté (Dumont, 2008).

Le second problème concerne le manque d'historicisation du « transnationalisme ». En effet, comparées aux thèses de la «fin des territoires » et du "post-nationalisme », les transnational studies semblent victimes d'un "présentisme », pour reprendre le mot de Roger Waldinger (2006) qui désigne ainsi la relative absence de réflexion des chercheurs sur la "présence du passé » au sein des réseaux transnationaux de migrants. Roger Waldinger souligne quatre modalités de l'articulation entre passé et présent et cite des travaux d'historiens établissant l'existence, dès le début du siècle, d'associations de migrants promouvant un nationalisme de longue distance (ibid. : 26). Prendre en compte cette épaisseur historique des associations de migrants nécessite donc de s'attarder sur leurs archives et de considérer les configurations historiques spécifiques des relations entre États d'origine et d'installation.

Enfin, le troisième problème touche à la dimension spatiale des phénomènes étudiés. Celle-ci est prise en compte dans les transnational studies, à propos des articulations entre l'échelle locale et l'échelle mondiale, mais l'espace y est le plus souvent défini comme support des réseaux sociaux. L'investissement de ce champ de recherches par les géographes se fonde au contraire sur une définition de l'espace comme élément constitutif de la transnationalisation elle-même : celle-ci engage des localisations et des configurations spatiales spécifiques et se distingue surtout par des spatialités et des territorialités qui entremêlent les échelles locales, nationales et mondiales (Jackson et al., 2003 : 1-2). Tenter d'approfondir cette piste implique d'intégrer à la réflexion les apports des travaux sur les champs et les circulations migratoires, afin de définir plus finement les identités collectives revendiquées par les associations de migrants et les formes socio-spatiales qu'elles revêtent. 


\section{CONCLUSION}

En s'achevant sur ces possibles convergences entre les études sur les associations de migrants et des grilles de lecture plus générales du monde contemporain, cet état des recherches a tenté de montrer le déclin d'un certain nationalisme méthodologique, celui présent dans le paradigme de l'intégration. Il a aussi tenté de souligner la montée des approches pluridisciplinaires, dans un champ d'étude dont l'intérêt principal est de travailler des notions-clés des études migratoires, tout en étant situé au cœur des interrogations actuelles sur la globalisation. Finalement, cet état des recherches aura révélé autant d'acquis solides que de pistes à explorer. La compréhension du rôle et du fonctionnement de ces associations s'est en effet affinée grâce à l'observation répétée des pratiques militantes et des activités concrètes, grâce aussi aux notions d'ethnicité, de médiation et de négociation identitaire, de réseaux et de stratégies. Et l'avenir de cet objet est prometteur, car chaque vague migratoire continuera de produire ses propres regroupements. Pour autant, les recherches à venir devront accorder toute leur place à l'approche comparative, dans le temps et l'espace, et tenter de concilier, à travers l'objet " association de migrants ", les apports de la sociologie de l'action collective et de la géographie des migrations. Ce sont notamment les liens entre pratiques militantes et pratiques circulatoires qui restent à explorer.

\section{Références bibliographiques}

ANDERSON Benedict (1998) Long distance nationalism, in Benedict Anderson Ed., The spectre of comparisons. Nationalism, Southeast Asia and the World, Londres-New York, Verso, pp. 58-74.

ANDERSON Benedict (2002) L'imaginaire national. Réflexions sur l'origine et l'essor du nationalisme, Paris, La Découverte, 212 p.

APPADURAI Arjun (2001) Après le colonialisme. Les conséquences culturelles de la globalisation, Paris, Payot, $322 \mathrm{p}$.

BADIE Bertrand (1995) La fin des territoires. Essai sur le désordre international et sur l'utilité sociale du respect, Paris, Fayard, 278 p.

BAILLET Dominique (1998) Le militantisme des jeunes d'origine maghrébine en région parisienne de 1973 à nos jours : le passage du politique à l'économique, Thèse de sociologie, EHESS, Paris, $527 \mathrm{p}$.

BASCH Linda, GLICK SCHILLER Nina and SZANTON BLANC Cristina (1994) Nations unbound. Transnational projects, post-colonial predicaments, and deterritorialized NationStates, Royaume-Uni, Gordon and Breach, 344 p.

BASTENIER Albert (2004) Qu'est-ce qu'une société ethnique ?, Paris, PUF, 346 p.

BATTEGAY Alain (1990) La déstabilisation des associations beurs et la montée de l'ethnicité, Les Annales de la Recherche Urbaine, 49, pp. 104-114.

BERTOSSI Christophe (2001) Les frontières de la citoyenneté en Europe. Nationalité, résidence, appartenance, Paris, L'Harmattan, $300 \mathrm{p}$.

BIRNBAUM Pierre (Éd.) (1997) Sociologie des nationalismes, Paris, PUF, 462 p.

BLANCHARD Pascal, BANCEL Nicolas et LEMAIRE Sandrine (Éds.) (2005) La fracture coloniale : la société française au prisme de l'héritage colonial, Paris, La Découverte, $315 \mathrm{p}$.

BORDES-BENAYOUN Chantal et SCHNAPPER Dominique (2006) Diasporas et nations, Paris, Odile Jacob, 255 p. 
BOUAMAMA Saï, CORDEIRO Albano et ROUX Michel (1992) La citoyenneté dans tous ses états : de l'immigration à la nouvelle citoyenneté, Paris, CIEMI-L'Harmattan, $361 \mathrm{p}$.

BOUAMAMA Saïd (1994) Dix ans de marches des Beurs. Chronique d'un mouvement avorté, Paris, Desclée de Brouwer, $230 \mathrm{p}$.

BOUBEKER Ahmed et ABDALLAH Mogniss H. (1993) Douce France : la saga du mouvement Beur 1983-1993, Paris, Quo Vadis, 107 p.

BOUBEKER Ahmed (2003) Les mondes de l'ethnicité. La communauté d'expérience des héritiers de l'immigration maghrébine, Paris, Balland, $361 \mathrm{p}$.

BRUNEAU Michel (2004) Diasporas et espaces transnationaux, Paris, Anthropos, 249 p.

CATANI Maurizio et PALIDDA Salvatore (Éds.) (1987) Le rôle du mouvement associatif dans l'évolution des communautés immigrées, Paris, FAS-DPM, 548 p.

CÉSARI Jocelyne (1998) Musulmans et républicains : les jeunes, l'Islam et la France, Bruxelles, Éditions Complexe, $166 \mathrm{p}$.

CHIVALLON Christine (2002) Construction d'une mémoire relative à l'esclavage et instrumentalisation politique : le cas des anciens ports négriers de Bordeaux et Bristol, Cahiers des Anneaux de la Mémoire, 4, pp. 177-202.

CORDEIRO Albano (1986) Enfermement et ouvertures : les associations portugaises en France, Paris, CEDEP, $126 \mathrm{p}$.

DAUM Christophe (1998) Les associations de Maliens en France. Migrations, développement et citoyenneté, Paris, Karthala, 253 p.

DE RUDDER Véronique, POIRET Christian et VOURC'H François (2000) L'inégalité raciste. L'universalité républicaine à l'épreuve, Paris, PUF, 213 p.

DEWITTE Philippe (Éd.) (1999) Immigration et intégration. L'état des savoirs, Paris, La Découverte, $423 \mathrm{p}$.

DI MEO Guy et BULEON Pascal (Éds.) (2005) L'espace social. Une lecture géographique des sociétés, Paris, Armand Colin, $304 \mathrm{p}$.

DIECKHOFF Alain et JAFFRELOT Christophe (Éds.) (2006) Repenser le nationalisme. Théories et pratiques, Paris, Presses de Science Po, 463 p.

DORAIS Louis-Jacques (2004) À propos des migrations transnationales : l'exemple des Canadiens d'origine vietnamienne, Revue Européenne des Migrations Internationales, 20 (3), pp. 49-73.

DUBET François (1987) La galère. Jeunes en survie, Paris, Fayard, 503 p.

DUMONT Antoine (2007) La marocanité associative en France. Militantisme et territorialité d'une appartenance ressentie à distance, Thèse de géographie, Université de Poitiers, $624 \mathrm{p}$.

DUMONT Antoine (2008) Representing voiceless migrants: Moroccan political transnationalism and Moroccan migrants' organizations in France, Ethnic and Racial Studies, 31 (4), pp. 792-811.

FAIST Thomas (2004) Transnational social spaces: agents, networks, and institutions, Burlington, Ashgate, $237 \mathrm{p}$.

FARET Laurent (2003) Les territoires de la mobilité. Migration et communautés transnationales entre le Mexique et les États-Unis, Paris, CNRS Éditions, 351 p.

FENNEMA Meindert and TILLIE Jean (2004) Do Immigrant Policies Matter? Ethnic Civic Communities and Immigrant Policies in Amsterdam, Liege and Zurich, in Rinus Penninx, Karen Kraal, Marco Martiniello and Steven Vertovec Eds., Citizenship in European Cities: Immigrants, Local Politics, and Integration Policies, Aldershot-Burlington, Ashgate, pp. 85-106.

GEISSER Vincent (1997) Ethnicité républicaine. Les élites d'origine maghrébine dans le système politique français, Paris, Presses de Science Po, $261 \mathrm{p}$.

GEISSER Vincent (2005) Ethnicité républicaine versus République ethnique ?, Mouvements, 38, pp. 19-25.

GELLNER Ernest (1989) Nations et nationalisme, Paris, Payot, 208 p.

GIRAUD Michel et MARIE Claude-Valentin (1987) Insertion et gestion socio-politique de l'identité culturelle : le cas des Antillais en France, Revue Européenne des Migrations Internationales, 3 (3), pp. 31-48. 
GLICK SCHILLER Nina (1997) The situation of transnational studies, Identities, 4, pp. 155-166. GLICK SCHILLER Nina and EUGENE FOURON Georges (2002) Georges Woke Up Laughing. Long Distance Nationalism and the Search for Home, Durham NC, Duke University Press, $324 \mathrm{p}$.

GLICK SCHILLER Nina (2004) Transnationality, in David Nugent et Joan Vincent Eds., $A$ Companion to the Anthropology of Politics, Malden, Blackwell, pp. 448-467.

GONIN Patrick (1997) D'entre deux territoires : circulations migratoires et développement entre le bassin du fleuve Sénégal et la France, Habilitation à diriger des recherches, Université des Sciences et Technologies de Lille, $880 \mathrm{p}$.

GOSSIAUX Jean-François (2002) Pouvoirs ethniques dans les Balkans, Paris, PUF, 217 p.

GUILLAUMIN Colette (1972) L'idéologie raciste. Genèse et langage actuel, Paris-La Haye, Mouton, $247 \mathrm{p}$.

GUILLON Michèle et TABOADA-LEONETTI Isabelle (1986) Le triangle de Choisy, un quartier chinois à Paris. Cohabitation pluri-ethnique, territorialisation communautaire et phénomènes minoritaires dans le 13 e arrondissement, Paris, CIEMI-L'Harmattan, $210 \mathrm{p}$.

HANNERZ Ulf (1996) Transnational connections, Londres, Routledge, $201 \mathrm{p}$.

HAUT CONSEIL À L'INTÉGRATION (2000) L'islam dans la République, Paris, La Documentation française, $204 \mathrm{p}$.

HILY Marie-Antoinette (1993) Les identités collectives à l'épreuve de l'émigration-immigration : le cas des Portugais en France, Thèse de sociologie, Université de Nice Sophia Antipolis, 393 p.

HILY Marie-Antoinette et RINAUDO Christian (2002) L'intégration en question : territoires et construction de groupes, in Gilles Ferréol Éd., Rapport à autrui et personne citoyenne, Villeneuve d'Ascq, Presses Universitaires du Septentrion, pp. 215-234.

HOBSBAWM Éric (1992) Nations et nationalisme depuis 1780 : programme, mythe, réalité, Paris, Gallimard, $247 \mathrm{p}$.

ION Jacques (1997) La fin des militants ?, Paris, Éditions de l'Atelier, 124 p.

JACKSON Peter, CRANG Phil and DWYER Claire Eds. (2003) Transnational spaces, Londres-New York, Routledge, $189 \mathrm{p}$.

JAZOULI Adil (1986) L'action collective des jeunes maghrébins en France, Paris, CIEMIL'Harmattan, $217 \mathrm{p}$.

JUTEAU-LEE Danielle (1983) La production de l'ethnicité ou la part réelle de l'idéel, Sociologie et Sociétés, 15 (2), pp. 39-54.

KASTORYANO Riva (2006) Vers un nationalisme transnational. Redéfinir la nation, le nationalisme et le territoire, Revue française de science politique, 56 (4), pp. 533-553.

KEPEL Gilles (1987) Les banlieues de l'Islam : naissance d'une religion en France, Paris, Seuil, $429 \mathrm{p}$.

KOOPMANS Rudi andt STATHAM Paul (2001) How national citizenship shapes transnationalism. A comparative analysis of migrant claims-making in Germany, Great Britain and the Netherlands, Revue Européenne des Migrations Internationales, 14 (2), pp. 63-100.

KOTLOK-PIOT Nathalie (1994) La communauté portugaise en France : espace et devenir, Thèse de géographie, Université de Toulouse 2 Le Mirail, 532 p.

LAVILLE Jean-Louis et al. (2001) Associations, démocratie et société civile, Paris, La Découverte, $220 \mathrm{p}$.

LEVEAU Rémy et WIHTOL DE WENDEN Catherine (Éds.) (1991) Modes d'insertion des populations de culture islamique dans le système politique français, Paris, FNSP-CERI, Convention MIRE n² 247/87, 265 p.

LEVEAU Rémy et WIHTOL DE WENDEN Catherine (Éds.) (1996) Associations créées dans les années 1980 par les jeunes militants issus de l'immigration : bilan de leurs activités et de l'engagement de leurs promoteurs, Paris, FNSP-CERI, Contrat FAS.

LEVITT Peggy (2001) The transnational villagers, Berkeley, University of California Press, 281 p. 
LÉVY Jacques et LUSSAULT Michel (Éds.) (2003) Dictionnaire de la géographie et de l'espace des sociétés, Paris, Belin, $1034 \mathrm{p}$.

MA MUNG Emmanuel (1992) Dispositif économique et ressources spatiales : éléments d'une économie de diaspora, Revue Européenne des Migrations Internationales, 8 (3), pp. 175-193.

MARTINIELLO Marco (1995) L'ethnicité dans les sciences sociales contemporaines, Paris, PUF, $125 \mathrm{p}$.

NEVEU Catherine (1993) Communauté, nationalité et citoyenneté. De l'autre côté du miroir : les Bangladeshis de Londres, Paris, Karthala, 399 p.

ONG Aihwa (1999) Flexible citizenship. The cultural logics of transnationality, Durham NC, Duke University Press, $322 \mathrm{p}$.

ORIOL Michel (1985) L'ordre des identités, Revue Européenne des Migrations Internationales, 1 (2), pp. 171-195.

ORIOL Michel et HILY Marie-Antoinette (1985) Les réseaux associatifs des immigrés en Europe occidentale, Poitiers, Études méditerranéennes, 85 p.

POINARD Michel (1991) Les Portugais dans l'émigration : une géographie de l'absence, Thèse de Lettres et Sciences Humaines, Université de Toulouse 2 Le Mirail, 803 p.

POIRET Christian (1997) Familles africaines en France. Ethnicisation, ségrégation et communalisation, Paris, CIEMI-L'Harmattan, 448 p.

PORTES Alejandro, GUARNIZO Luis Eduardo and LANDOLT Patricia (1999) Introduction: pitfalls and promise of an emergent research field, Ethnic and Racial Studies, 22 (2), pp. 217-237.

POUTIGNAT Philippe et STREIFF-FENART Jocelyne (1995) Théories de l'ethnicité, Paris, PUF, $270 \mathrm{p}$.

QUIMINAL Catherine (1991) Gens d'ici, gens d'ailleurs. Migrations Soninké et transformations villageoises, Paris, Christian Bourgeois Éditeur, 223 p.

RINAUDO Christian (2000) L'ethnicité dans la cité. Jeux et enjeux de la catégorisation ethnique, Paris, L'Harmattan, 206 p.

SIMÉANT Johanna (1998) La cause des sans-papiers, Paris, Presses de Science Po, 504 p.

SIMON Patrick (2003) Les sciences sociales françaises face aux catégories ethniques et raciales, Annales de Démographie Historique, 1, pp. 111-130.

SIMON Pierre-Jean (1994) Ethnicité, Pluriel Recherches : Vocabulaire Historique et Critique des Relations Inter-ethniques, 2, pp. 14-20.

SMITH Michael Peter and GUARNIZO Luis Eduardo (1998) Transnationalism from below, New Brunswick, Transaction, $316 \mathrm{p}$.

SMITH Robert (2001) Migrant Membership as an Instituted Process: Comparative Insights from the Mexican and Italian Cases, Oxford, ESRC (voir http://www.transcomm.ox.ac.uk/working\%20 papers/WPTC-01-23\%20Smith.pdf).

SOYSAL Yasemin Nuhoglu (1994) Limits of Citizenship: Migrants and Postnational membership in Europe, Chicago, University of Chicago Press, 244 p.

SRAIEB Nourreddine et al. (1985) Le mouvement ouvrier maghrébin, Paris, CNRS, 327 p.

TIMERA Mahamet (1997) L'immigration africaine en France : regards des autres et repli sur soi, Politique Africaine, 67, pp. 40-47.

TOURAINE Alain, WIEVIORKA Michel et DUBET François (1984) Le mouvement ouvrier, Paris, Fayard, 438 p.

VERBUNT Gilles (1980) L'intégration par l'autonomie. La CFDT, l'Église catholique, la FASTI face aux revendications d'autonomie des travailleurs immigrés, Paris, CIEMM, 398 p.

VERTOVEC Steven (1999) Conceiving and Researching Transnationalism, Ethnic and Racial Studies, 22 (2), pp. 447-462.

WALDINGER Roger (2006) «Transnationalisme » des immigrants et présence du passé, Revue Européenne des Migrations Internationales, 22 (2), pp. 23-41.

WEBER Max (1971) Économie et société, Paris, Plon, 650 p. 
WEILL Claudie (1981) Territoire et extra-territorialité : les théories socialistes des empires multinationaux avant 1914, Pluriel Débat, 26, pp. 39-44.

WEILL Claudie (1987) L'Internationale et l'autre. Les relations inter-ethniques dans la IIe Internationale, Paris, Arcantère, $163 \mathrm{p}$.

WIHTOL DE WENDEN Catherine (1988) Les immigrés et la politique, Paris, Presses de la FNSP, $393 \mathrm{p}$.

WIHTOL DE WENDEN Catherine et LEVEAU Rémy (2001) La beurgeoisie. Les trois âges de la vie associative issue de l'immigration, Paris, CNRS Éditions, 188 p. 


\title{
L'état des recherches sur les associations de migrants
}

\author{
Antoine DUMONT
}

Cet article se propose de retracer les évolutions de la recherche française sur les associations de migrants internationaux, à partir d'une revue critique des travaux réalisés ces vingt dernières années. Centrées au départ sur la notion d'intégration, ces recherches ont progressivement adopté le paradigme de l'ethnicité, puis celui du « transnationalisme», en s'inspirant des travaux anglo-saxons et européens. L'approche proposée ici se veut critique, au sens où ces trois paradigmes ne permettent pas toujours de saisir l'ensemble des significations que les migrants accordent à leur origine nationale, lorsque celle-ci est le moteur de leur engagement associatif. Désigner cet ensemble de significations par le terme de «nationité associative » permet à l'inverse de combler quelques-unes des lacunes repérées, tout en maintenant ouvert le cadre interprétatif.

\section{Migrants Associations. A State of the Art}

\section{Antoine DUMONT}

This article suggests going back to the evolution of the French research on the international migrant associations, examining with a critical eye the works that have been carried out over these past twenty years. Focusing first on the notion of integration, these researches gradually adopted the ethnicity then the transnational paradigms, inspired by Anglo-Saxon and European works. The approach proposed here is critical, in the sense that these three paradigms do not always enable one to grasp all the meanings that the migrants grant to their national origin, when the latter is the mainspring of their associative commitment. Naming this set of meanings by the term of "associative nationness" allows to fill some of the gaps that have been noticed, while maintaining an interpretative frame open.

\section{El estado de las búsquedas sobre las asociaciones de emigrantes}

\section{Antoine DUMONT}

Este artículo propone volver a trazar las evoluciones de la búsqueda francesa sobre las asociaciones de emigrantes internacionales, a partir de una lectura crítica de los trabajos realizados estos últimos veinte años. Centradas al principio sobre la noción de integración, estas búsquedas progresivamente adoptaron el paradigma de la etnicidad, luego él del transnacionalismo, inspirándose en trabajos anglosajones y europeos. El enfoque propuesto aquí se considera crítico, en el sentido en que estos tres paradigmas no permiten siempre captar el conjunto de los significados que los emigrantes otorgan a su origen nacional, cuando éste es el motor de su compromiso asociativo. Designar este conjunto de significados por el término de «naciondad asociativo» permite colmar algunos huecos en los que se ha reparado, manteniendo abierto el marco interpretativo. 